\title{
The vertebrate makorin ubiquitin ligase gene family has been shaped by large-scale duplication and retroposition from an ancestral gonad-specific, maternal-effect gene
}

Astrid Böhne ${ }^{1}$, Amandine Darras $^{1 \dagger}$, Helena D'Cotta ${ }^{2 \dagger}$, Jean-Francois Baroiller ${ }^{2}$, Delphine Galiana-Arnoux ${ }^{1}$, Jean-Nicolas Volff ${ }^{*}$

\begin{abstract}
Background: Members of the makorin (mkrn) gene family encode RING/C3H zinc finger proteins with U3 ubiquitin ligase activity. Although these proteins have been described in a variety of eukaryotes such as plants, fungi, invertebrates and vertebrates including human, almost nothing is known about their structural and functional evolution.

Results: Via partial sequencing of a testis CDNA library from the poeciliid fish Xiphophorus maculatus, we have identified a new member of the makorin gene family, that we called mkrn4. In addition to the already described $m k r n 1$ and $m k r n 2, m k r n 4$ is the third example of a makorin gene present in both tetrapods and ray-finned fish. However, this gene was not detected in mouse and rat, suggesting its loss in the lineage leading to rodent murids. Mkrn2 and mkrn4 are located in large ancient duplicated regions in tetrapod and fish genomes, suggesting the possible involvement of ancestral vertebrate-specific genome duplication in the formation of these genes. Intriguingly, many $m k r n 1$ and $m k r n 2$ intronless retrocopies have been detected in mammals but not in other vertebrates, most of them corresponding to pseudogenes. The nature and number of zinc fingers were found to be conserved in Mkrn1 and Mkrn2 but much more variable in Mkrn4, with lineage-specific differences. RT-qPCR analysis demonstrated a highly gonad-biased expression pattern for makorin genes in medaka and zebrafish (rayfinned fishes) and amphibians, but a strong relaxation of this specificity in birds and mammals. All three mkrn genes were maternally expressed before zygotic genome activation in both medaka and zebrafish early embryos.

Conclusion: Our analysis demonstrates that the makorin gene family has evolved through large-scale duplication and subsequent lineage-specific retroposition-mediated duplications in vertebrates. From the three major vertebrate $m k r n$ genes, $m k r n 4$ shows the highest evolutionary dynamics, with lineage-specific loss of zinc fingers and even complete gene elimination from certain groups of vertebrates. Comparative expression analysis strongly suggests that the ancestral E3 ubiquitin ligase function of the single copy $m \mathrm{krn}$ gene before duplication in vertebrates was gonad-specific, with maternal expression in early embryos.
\end{abstract}

\footnotetext{
* Correspondence: Jean-Nicolas.Volff@ens-lyon.fr

+ Contributed equally

'Institut de Génomique Fonctionnelle de Lyon, Université de Lyon, Université Lyon 1, CNRS, INRA, Ecole Normale Supérieure de Lyon, Lyon, France

Full list of author information is available at the end of the article
} 


\section{Background}

Despite their presence in organisms as diverse as fungi, plants and animals, the functions and evolution of Makorin (Mkrn) proteins in eukaryotes remain poorly understood. Makorins are zinc finger proteins with a typical C3HC4 motif called the RING domain. This protein-protein interaction motif is found in most E3 ubiquitin ligases, a category of enzymes mediating the transfer of ubiquitin from an E2 ubiquitin-conjugating enzyme to target protein substrates. The RING domain is responsible for the ubiquitin ligase activity, leading to mono-ubiquitination and/or to synthesis of poly-ubiquitin chains on lysine residues (for review [1]). Accordingly, some Makorin proteins work as E3 ubiquitin ligases [2]. In Makorin proteins, the RING domain is associated with typical arrays of one to four $\mathrm{C} 3 \mathrm{H}$ domains, a type of zinc finger found in a variety of ribonucleoproteins [3]. Another motif rich in Cys and His residues, with so far unknown function, is also generally present in Mkrn proteins [4](Figure 1).

Three functional $m k r n$ genes, $m k r n 1,2$ and 3, have been described so far in vertebrates, with $m k r n 3$ being specific to therian mammals. Makorin1 has been well studied in mammals. Its described functions are linked to E3 ubiquitin ligase activity, for example ubiquitination-mediated degradation of capsid proteins as a defence mechanism against virus infection [5]. Mkrn1 is also involved in the control of cell cycle arrest and apoptosis through ubiquitination and proteasomedependent degradation of proteins p53 and p21 [6]. Mkrn1 targets human telomerase catalytic subunit (hTERT) for proteasome processing during differentiation or cell cycle arrest [7]. Makorin1 might also have transcriptional activity and regulates RNA polymerase II-dependent transcription independently from E3 ligase activity, with either negative or positive effects on gene expression [8]. Changes in $m k r n 1$ expression have been associated with different types of cancer and other diseases, as observed for other RING proteins with E3 ligase activity [9-12].

A close relative to $m k r n 1$ is $m k r n 3$ (aka $Z n f 127$ in human and mouse), which has been detected only in therian mammals. Mkrn3 has been first identified in the Prader-Willi syndrome critical region on $15 \mathrm{q} 11.2$ in the human genome $[13,14]$, but with no obvious role in the disease [15]. No function has been assigned so far to

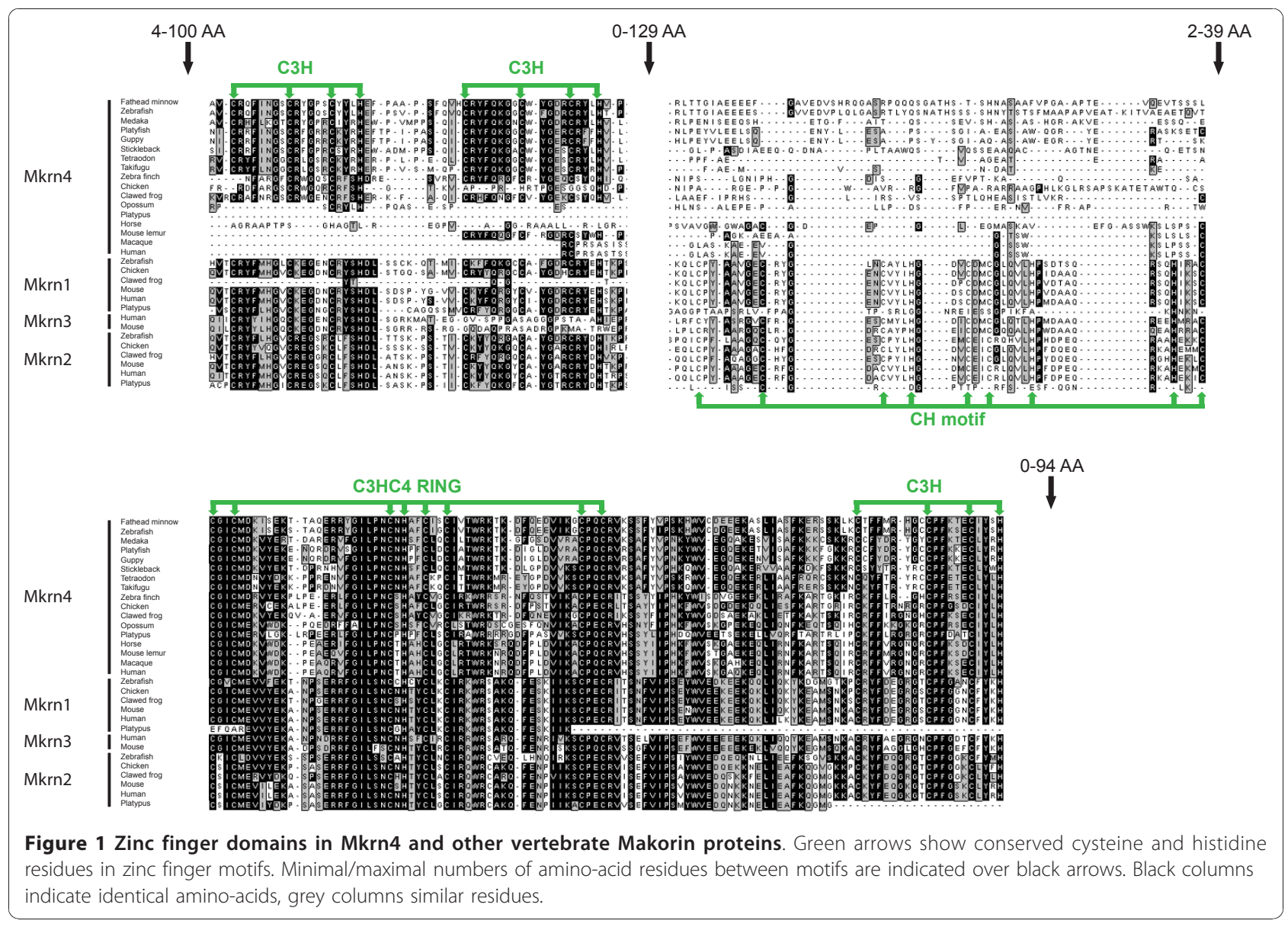


Mkrn3. Mkrn3 in fact corresponds to an intronless retrocopy of $m k r n 1$ generated through reverse transcription of an $m k r n 1$ mRNA molecule. The formation of such retrogenes is catalyzed by the reverse transcriptase encoded by autonomous retrotransposable elements. Several other retrocopies of $m k r n 1$ have been identified in mammalian genomes, most of them probably corresponding to pseudogenes. The possible involvement of such pseudogenes in the regulation of the founding source gene $m k r n 1$ has been proposed and debated $[8,13,14,16]$.

The third functional member of the makorin family described in vertebrates is $m k r n 2$. This gene partially overlaps with the raf1 proto-oncogene in antisense transcriptional orientation [17]. Functional data have been only published for the clawed frog Xenopus laevis, where Mkrn2 is a neurogenesis inhibitor acting upstream of glycogen synthase kinase-3beta (GSK-3beta) in the phosphatidylinositol 3-kinase/Akt pathway. The third $\mathrm{C} 3 \mathrm{H}$ zinc finger, the Cys-His motif as well as the RING zinc finger are necessary for this anti-neurogenic activity $[18,19]$.

During the screening of a testis cDNA library from the platyfish Xiphophorus maculatus (poeciliid), we have identified a gene with gonad-specific expression representing a new divergent member of the makorin gene family in vertebrates, which was named mkrn4. Comparative analyses in different species revealed the evolutionary dynamics of this family of RING finger proteins, suggesting an ancestral gonad-specific function and maternal embryonic expression before duplication in vertebrates.

\section{Results and discussion}

Mkrn4 is a new member of the vertebrate makorin gene family

In order to identify new potential gonad-specific transcription factors or regulatory proteins expressed in fish gonads, we randomly sequenced clones from a testis cDNA library of the platyfish Xiphophorus maculatus. In silico screening for sequences containing zinc finger domains revealed a clone with similarity to uncharacterized members of the makorin gene family [20] (Genbank accession number HQ377193). Since three functional $m k r n$ genes have been described so far in vertebrates, the newly identified gene was called makorin4 (mkrn4).

We could identify sequences orthologous to platyfish $m k r n 4$ in other fish species (Figures 1 and 2). All fully sequenced fish genomes including zebrafish (Danio rerio), medaka (Oryzias latipes [21], green spotted pufferfish (Tetraodon nigroviridis),[22], fugu (Takifugu rubripes) [23] and three-spined stickleback (Gasterosteus aculeatus) contained $m k r n 4$ as a single copy gene. Mkrn4 ESTs were also identified in databases for other fish species without available genome draft. $M k r n 4$ is present in birds (on Gga26 in chicken) and amphibians as well as in

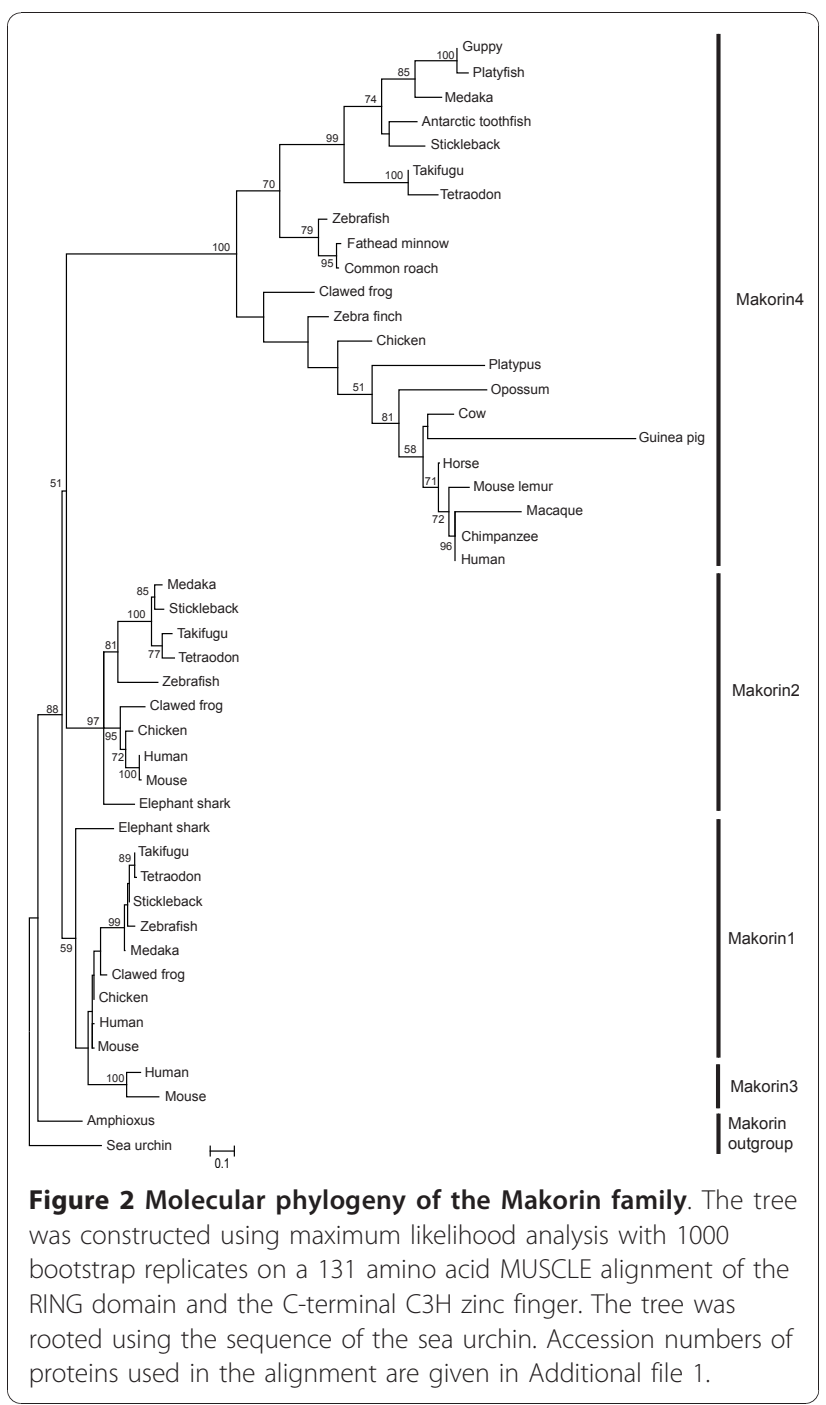

most mammals, including human (Hsa6q14.1, where it was annotated as a pseudogene, see below). Mkrn4 sequences form a clear monophyletic group within the Makorin molecular phylogeny (Figure 2).

Surprisingly, analysis of genome sequence drafts and EST data failed to detect mkrn4 in two murid rodents, the mouse Mus musculus and rat Rattus norvegicus, even after targeted scrutiny of chromosomal segments syntenic to $m k r n 4$-containing loci from other species (on Mmu17 in mouse and Rno20 in rat). Partial mkrn4 sequences were found in other rodents such as Ord's kangaroo rat (Dipodomys ordii), thirteen-lined ground squirrel (Spermophilus tridecemlineatus) and Guinea pig (Cavia porcellus; for this species a deduced sequence could be included into the Mkrn phylogeny), as well as in the American pika Ochotona princeps (lagomorph). No $m k r n 4$-related sequences were identified in the genome draft of another lagomorph, the rabbit Oryctolagus cuniculus, and in the northern tree shrew Tupaia belangeri 
(order Scandentia). For all these species, no $m k r n 4$ EST sequence was present in databases. Hence, these results suggest that the $m k r n 4$ gene has been lost in murid rodents (mouse and rat). In some other rodent or lagomorph species, mkrn4 may be also on the way of degradation (as suggested by long branch for Guinea pig sequence; Figure 2) or even might have been already eliminated, but genomic sequences of better quality will be required to confirm this hypothesis.

We noticed that mkrn4 was annotated as a pseudogene in the current Ensembl human genome release, under the name of Mkrnp2 [24]. Very similar orthologous sequences were identified in syntenic regions of the genome of chimpanzee (chr. 6, 99\% nucleotide identity with the human sequence in the coding region), orangutan (chr. 6, 99\% nucleotide identity) and macaque (chr. 4, 96\% nucleotide identity), a degree of conservation surprising for a pseudogene evolving under neutral selection. Only gorilla did not show any $m k r n 4$ sequence, despite the presence of a large syntenic region on chromosome 6 including several genes surrounding $m k r n 4$ in other primates. However, the draft sequence of this region contains many gaps, especially at the position where $m k r n 4 / m k r n p 2$ would be expected. Hence, $m k r n 4$ might be absent from draft but present in the genome of the gorilla.

In human, mkrnp2 was annotated as pseudogene due to a premature stop codon at position 53 in the Ensembl transcript. We suggest that the translation start codon was wrongly annotated in the Ensembl prediction. A start codon in frame +3 , using the ATG at position 54 of the predicted transcript, leads to a full length protein without any further interruption of the open reading frame. This ATG is conserved between primate sequences and annotated as the bona fide start codon in GenBank entry EAX03829.1 used in sequence alignments and molecular phylogenies (Figures 1 and 2). Primate sequences were reannotated accordingly (Figure 1).

Using primate sequences as query against EST databases in BLAST analysis, no evidence of transcription could be found. We then compared the rate of nonsynonymous $(\mathrm{Ka})$ to the rate of synonymous substitutions (Ks) between $m k r n$ sequences in primates (human, chimpanzee, orangutan and macaque) and other vertebrates. The average $\mathrm{Ka} / \mathrm{Ks}$ value obtained between primate $m k r n 4$ sequences was 0.12 , indicating an excess of synonymous vs. non-synonymous substitutions. This value was similar to those obtained between primate $m k r n 1(0.16)$ and $m k r n 2$ (0.11). The average $\mathrm{Ka} / \mathrm{Ks}$ ratio for $m k r n 4$ between fish sequences (medaka, platyfish and guppy) was 0.27 . Taken together, these observations suggest that $m k r n 4$ evolved under purifying selection in primates and fish, as observed for other makorin genes. Mkrn4 might be a functional protein-coding gene in primates but expressed at a very low level, in a particular type of cells or under very specific conditions. Alternatively, $m k r n 4$ might have lost its expression potential after initial functionality in different primate species.

No $m k r n 4$ sequence was found in the genome of more divergent species, including the elephant shark Callorhinchus milii (Chondrichthyes aka cartilaginous fish, a divergent jawed vertebrate) [25] and the invertebrate chordates Branchiostoma floridae (amphioxus) and Ciona intestinalis/savignyi (sea squirts).

\section{Large-scale duplication and the evolution of the makorin gene family}

Mkrn1, $m k r n 2$ and $m k r n 4$ are all present in both rayfinned fish and tetrapod lineages, indicating that these genes have been formed through gene duplications at least 450 million years ago early in the vertebrate lineage. Mkrn 1 and $m k r n 2$ but not $m k r n 4$ were detected in the genome of elephant shark Callorhinchus milii, a cartilaginous fish. In contrast, we could identify only one makorin gene in amphioxus and sea squirts (non-vertebrate chordates), as well as in other more divergent non-vertebrate species including yeast and rice. In the fly Drosophila melanogaster, four makorin genes were identified, but three of them clearly corresponded to retrocopies of the only bona fide intron-containing $m \mathrm{krn}$ gene. Hence, the $m k r n$ gene family observed in vertebrates is probably the result of two events of gene duplication having taken place between the emergence of vertebrates and the divergence between tetrapods and ray-finned fish. At least the duplication having led to $m k r n 1$ and $m k r n 2$ occurred before the split between cartilaginous and bony fish.

In order to know if gene duplications at the origin of the $m k r n$ family might be linked to rounds of whole genome duplications having occurred at the basis of the vertebrate lineage [26-29], we tested if $m k r n$ genes might be included in large ancient duplicated regions called paralogons having resulted from such events.

On human chromosome $3, m k r n 2$ and the raf1 protooncogene partially overlap in antisense transcriptional orientation. This syntenic relationship is conserved in most vertebrates but not in teleost fish, with the exception of the zebrafish, where $m k r n 2$ and raf1 are closely linked on chromosome 11 (Figure 3). Strikingly, in medaka raf1 is located close to $m k r n 4$ but not $m k r n 2$. We further analyzed syntenic relationships between genomic regions containing $m k r n 2$ and $m k r n 4$ in human, chicken, medaka and zebrafish genomes (Figure 3 ). We could identify a set of genes with duplicated copies linked to $m k r n 2$ and $m k r n 4$ in at least one species (for example ppard/pparg in human, chicken and medaka). Other genes like raf1 or $c n p b$ showed only one copy but either linked to $m k r n 2$ in some species or 


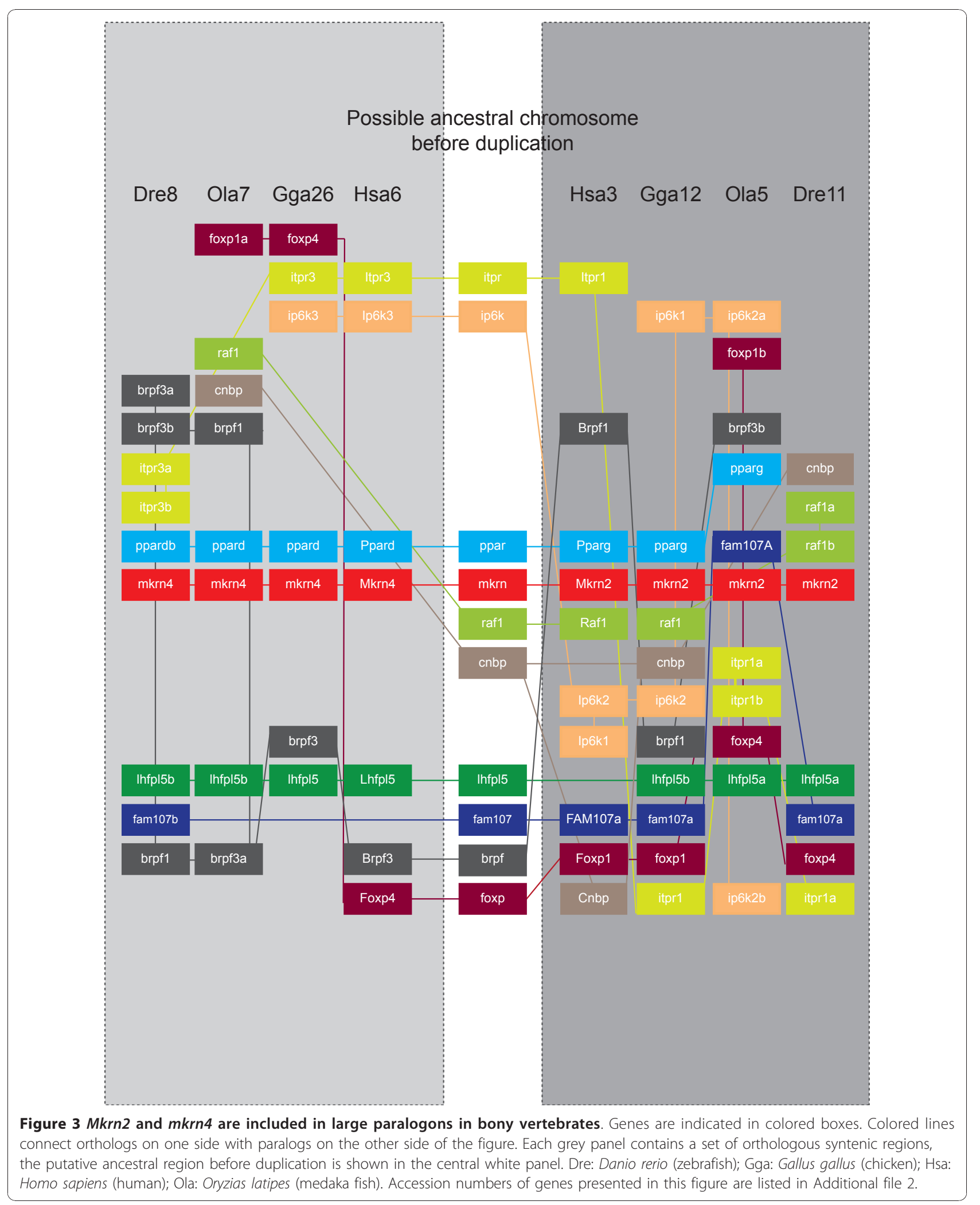


to $m k r n 4$ in others, suggesting differential loss of paralogous copies after duplication.

These results indicate that $m k r n 2$ and $m k r n 4$ are parts of ancient large duplicated regions present in distant vertebrate species. Hence, these paralogons might have been generated by one of the whole genome duplications having occurred in the ancestral vertebrate lineage, the so called $1 \mathrm{R}$ and $2 \mathrm{R}$ duplications [26-29]. Both $m k r n 2$ and $m k r n 4$ are called ohnologs (paralogous sequences resulting from whole genome duplications). On the other hand, we can not exclude the occurrence of a very large segmental gene duplication having generated these duplicated blocks.

The region containing $m k r n 1$ shows little conservation between mammals and fish (data not shown). We could not find any evidence for synteny between the mkrn1containing region and other $m k r n$ regions. It has been previously proposed that $m k r n 1$ and $m k r n 2$ are indeed the result of a single gene duplication event [17].

\section{Retroposition and the evolution of the makorin gene family}

In addition to $m k r n 1, m k r n 2$ and $m k r n 4$, multiple other $m k r n$-like sequences are present in mammalian genomes. Some of these sequences correspond to intronless retrocopies of $m k r n 1$. Their functional status concerning expression, regulation and mutual interference has been the source of controversial debate [16,30].

In order to identify all vertebrate $m k r n$-related sequences, we searched over the Ensembl Genome Browser the current genome releases of representatives of all vertebrate groups including all mammalian genomes [24]. The great majority of identified sequences corresponded to retrogenes of mkrn1 (Table 1), with the characteristic absence of introns due to reverse transcription of the mature mRNA molecule [31]. Besides $m k r n 3$, which probably corresponds to an expressed functional retrogene present in human and mouse, as many as ten different retrogenes located on eight different chromosomes were identified in the human genome. Most of them presented inactivating mutations or severely truncated open reading frames. Almost no EST corresponding to these retrogenes was detected in databases. Hence, most of these $m k r n 1$ retrocopies probably correspond to pseudogenes. Six $m k r n 1$ retropseudogenes located on five different chromosomes were identified in the mouse.

We detected makorin3 and other retrocopies of makorin1 only in mammals and not in chicken, frog and all five fish genomes analyzed. An mkrn3 sequence was detected in opossum, suggesting an event of retroposition that took place before the split of marsupials from therian mammals. No mkrn3 was found in the current genome draft of the platypus.
Retrocopies of $m k r n 2$ were found in the mouse but neither in the human nor in non-mammalian vertebrate genomes (Table 1). These three retropseudogenic copies of $m k r n 2$ are located on three different chromosomes in M. musculus. No retrocopy of $m k r n 4$ was found in all vertebrate genomes analysed, but three potentially functional retrocopies of the single intron-containing $m \mathrm{krn}$ gene were identified in the fruit fly Drosophila melanogaster. Taken together, these observations demonstrate an intriguing tendency of the $m k r n$ gene family to generate duplicated copies by retroposition.

\section{Structural evolutionary dynamics in the Makorin family}

The evolution of the nature and number of zinc fingers in the Makorin protein family was investigated (Figure 4). In most plants and invertebrate species (but not in Drosophila), the single Makorin protein contains the RING domain flanked by three $\mathrm{C} 3 \mathrm{H}$ zinc fingers on its $\mathrm{N}$-terminal side and one $\mathrm{C} 3 \mathrm{H}$ zinc finger on its $\mathrm{C}$-terminal side. An additional motif rich in cysteine and histidine residues (the CysHis motif) is located between the third $\mathrm{C} 3 \mathrm{H}$ zinc finger and the RING domain (Figures 1 and 4). This structure, likely to be ancestral in vertebrates, is also found in most, if not all Mkrn1 and Mkrn2 proteins.

In contrast, this otherwise well conserved structure was not found in Mkrn4 proteins (Figures 1 and 4). While the RING domain and the $\mathrm{C}$-terminal $\mathrm{C} 3 \mathrm{H}$ zinc finger are found in all Mkrn4 sequences, the third $\mathrm{C} 3 \mathrm{H}$ zinc finger and the CysHis motif are missing and have been probably lost in the ancestral mkrn4 gene early after duplication.

Only fish and Xenopus have kept the first two $\mathrm{C} 3 \mathrm{H}$ complete domains, which are both absent from mammalian sequences. In birds, a remnant of one of these domains has been retained in chicken and zebra finch under the form of a $\mathrm{C} 2 \mathrm{H}$ domain, the second $\mathrm{C} 3 \mathrm{H}$ domain having been maintained only in zebra finch but not in chicken.

These results indicate that the evolution of the Mkrn4 protein is associated with the dynamic loss of zinc finger domains otherwise conserved in Mkrn proteins. This is consistent with a to some extent faster evolution also reflected by the longer branches in the Makorin-like group in the phylogenetic tree (Figure 2). Another example of zinc finger domain loss is visible in $m k r n 3$ : this $m k r n 1$-derived retrogene encodes a protein having lost the second $\mathrm{C} 3 \mathrm{H}$ zinc finger (Figures 1 and 3 ) in human and mouse.

In the much conserved Mkrn1 and Mkrn2 groups, the platypus sequences present a reduced number of zinc fingers, like the opossum Mkrn2 sequence. This might reflect a biological reality, but might also be an artifact of local poor sequence assembly quality of the genome drafts for these species. 
Table 1 Mkrn1- and mkrn2-related retro(pseudo)genes in human and mouse genomes

\begin{tabular}{|c|c|c|c|}
\hline Location & Gene names & Annotation & EST support \\
\hline \multicolumn{4}{|l|}{ Human mkrn 1} \\
\hline Hsa7 140.152.840-140.179.369 & MKRN1, RNF61, ENSG00000133606 & $\begin{array}{l}\text { source protein-coding } \\
\text { gene with introns }\end{array}$ & expressed in many adult tissues \\
\hline Hsa15 23.810.454-23.813.164 & $\begin{array}{l}\text { MKRN3, D15S9, MGC88288, RNF63, } \\
\text { ZFP127, ZNF127 }\end{array}$ & protein-coding retrogene & $\begin{array}{l}\text { EST data for blood, brain, esophagus, liver, placenta, } \\
\text { prostate and skin }\end{array}$ \\
\hline Hsa1 43.356.765-43.358.101 & $\begin{array}{l}\text { ENSG00000237090, RP11-342M1.6, } \\
\text { MKRNP4* }\end{array}$ & retropseudogene & no EST support \\
\hline Hsa2 153.707.170-153.707.338 & ENSG00000234932 & retropseudogene & no EST support \\
\hline Hsa3 130.647.120-130.646.607 & $\begin{array}{l}\text { located in intron of ATP2C1 } \\
\text { (ENSG00000017260), MKRNP5* }\end{array}$ & retropseudogene & no EST support \\
\hline Hsa9 99.488.172-99.489.449 & $\begin{array}{l}\text { RP11-535M15.2, ENSG00000233820, } \\
\text { makorin ring finger protein } 1 \\
\text { pseudogene }\end{array}$ & retropseudogene & no EST support \\
\hline Hsa12 88.177.249-88.178.345 & $\begin{array}{l}\text { MKRN9P MKRNP6, MKRN5, MKRN9, } \\
\text { RNF65, ZNF127L3, ENSG00000213267 }\end{array}$ & retropseudogene & no EST support \\
\hline Hsa12 88.176.895-88.176.996 & ENSG00000237340 & retropseudogene & Hypothalamus Bl457939.1 \\
\hline Hsa20 45.092.541-45.093.763 & $\begin{array}{l}\text { MKRNP3, ENSG00000225849, RP5- } \\
\text { 981L23.2, DB295917.1 }\end{array}$ & retropseudogene & no EST support \\
\hline HsaX 40.693.508-40.697.060 & $\begin{array}{l}\text { MKRNP5, MKRN4, ZNF127-Xp, } \\
\text { ZNF127L1, ZNF-Xp, ENSG00000238222, } \\
\text { OTTHUMG00000024109, }\end{array}$ & $\begin{array}{l}\text { partially processed } \\
\text { retropseudogene }\end{array}$ & no EST support \\
\hline HsaX 73.380.895-73.382.363 & $\begin{array}{l}\text { MKRNP1, ZNF127-Xq, RP13-204A15.3, } \\
\text { ENSG00000224430 }\end{array}$ & retropseudogene & no EST support \\
\hline HsaX 91.627.539-91.627.653 & inside intron of $P C D H 11 X$ & $\begin{array}{l}\text { retro(pseudo?)gene, very } \\
\text { short but intact ORF }\end{array}$ & no EST support \\
\hline HsaY 5.352.120-5.352.234 & inside intron of $P C D H 11 Y$ & $\begin{array}{l}\text { retro(pseudo?)gene, very } \\
\text { short but intact ORF }\end{array}$ & no EST support \\
\hline \multicolumn{4}{|l|}{ Mouse mkrn1 } \\
\hline Mmu6 39.347.803-39.370.437 & $\begin{array}{l}\text { Mkrn1, ENSMUSG00000029922, } \\
\text { NM_018810 }\end{array}$ & $\begin{array}{l}\text { source protein-coding } \\
\text { gene with introns }\end{array}$ & $\begin{array}{l}\text { widely expressed in adult tissue with strong } \\
\text { expression in adult gonad and embryonic nervous } \\
\text { system [ref. [30] and EST data] }\end{array}$ \\
\hline Mmu7 69.563.293-69.564.927 & Mkrn3, ENSMUSG00000070527 & protein-coding retrogene & $\begin{array}{l}\text { EST data for brain, inner ear, pancreas, spinal cord } \\
\text { and thymus }\end{array}$ \\
\hline Mmu2 27.338.749-27.339.289 & no annotation & $\begin{array}{l}\text { newly annotated } \\
\text { retropseudogene }\end{array}$ & no EST support \\
\hline $\begin{array}{l}\text { Mmu3 129.843.302- } \\
\text { 129.845.025 }\end{array}$ & no annotation & retropseudogene & no EST support \\
\hline Mmu5 89.267.034-89.268.427 & $\begin{array}{l}\text { Mkrn1-ps1, AC160533.6, } \\
\text { ENSMUSG00000082389, AF494488 }\end{array}$ & retropseudogene & no EST support \\
\hline $\begin{array}{l}\text { Mmu5 111.334.263- } \\
111.334 .760\end{array}$ & $\begin{array}{l}\text { AC121934.4 (ENSMUSG00000081104), } \\
\text { OTTMUSG00000028665 }\end{array}$ & retropseudogene & no EST support \\
\hline Mmu13 77.911.041-77.911.846 & $\begin{array}{l}\text { in Intron of 1110033M05Rik } \\
\text { (ENSMUSG00000064138) }\end{array}$ & retropseudogene & no EST support \\
\hline MmuX 54.923.521-54.923.664 & $\begin{array}{l}\text { RP23-435H13.3, } \\
\text { OTTMUSG00000017520 }\end{array}$ & retropseudogene & no EST support \\
\hline \multicolumn{4}{|l|}{ Mouse $m k r n 2$} \\
\hline $\begin{array}{l}\text { Mmu6 115.551.979- } \\
115.568 .688\end{array}$ & $\begin{array}{l}\text { Mkrn2, ENSMUSG00000000439, } \\
\text { C81377; 2610002L04Rik }\end{array}$ & $\begin{array}{l}\text { source protein-coding } \\
\text { gene with introns }\end{array}$ & EST data for several tissue \\
\hline Mmu10 30.093.050-30.095.334 & no annotation & retropseudogene & no EST support \\
\hline MmuX 13.504.413-13.506.637 & $\begin{array}{l}\text { BX005215.13, ENSMUSG00000082736, } \\
\text { RP23-280F9.1, OTTMUSG00000016911 }\end{array}$ & $\begin{array}{l}\text { Partially processed } \\
\text { retropseudogene } \\
\text { (contains only } 2 \text { introns) }\end{array}$ & no EST support \\
\hline $\begin{array}{l}\text { Mmu2 106.753.075- } \\
106.754 .515\end{array}$ & no annotation & retropseudogene & no EST support \\
\hline
\end{tabular}

Positions are based on human GRCh37 and mouse NCBIM37 assemblies. Gene names marked with "*" refer to reference [4]. 


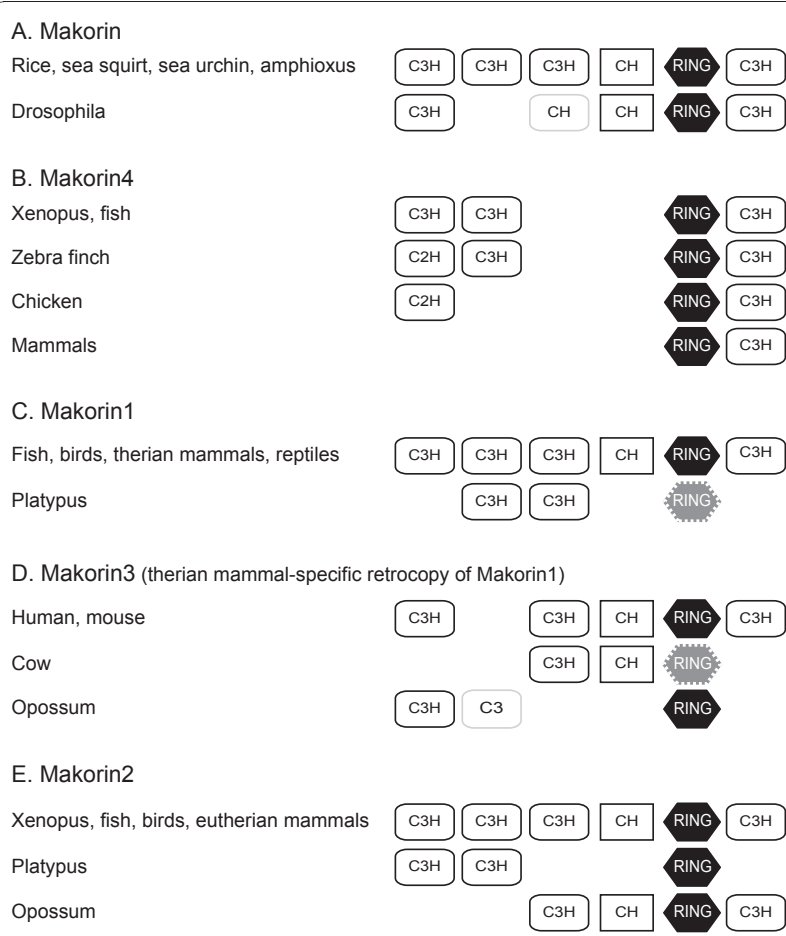

Figure 4 Zinc finger domains in the Makorin protein family. Black hexagons indicate the RING domain, white rectangles the $\mathrm{C} 3 \mathrm{H}$ zinc fingers. Dashed lines and lighter content indicate incomplete motifs due to sequence changes or loss. The structure of Mkrn2 for Xenopus is based on a X. laevis EST (EF626804) since the tropicalis sequence is incomplete and lacks the RING domain. Accession numbers are provided in Additional file 2.

\section{Evolution of makorin expression in vertebrates}

We compared the expression of the different vertebrate makorin genes in different species representative of the fish (medaka and zebrafish), amphibian (the African clawed frog Xenopus laevis) and bird (chicken) lineages (published and EST data are generally available for mammals) (Figures 5 and 6). This might allow to assess the functional evolution of $m k r n$ genes in vertebrates and to estimate the ancestral expression pattern and function in the primitive vertebrate before gene duplication.

In adult tissue, the whole $m k r n$ gene family showed a clear gonad-biased expression in fish and frog, with particular high expression in ovary (Figure 5). Accordingly, $m k r n 1$ is upregulated during ovarian maturation in flatfish, with strong expression in previtellogenic ovarian follicles [32]. Such a specificity of expression was not observed in birds and mammals, even if $m k r n 1$ has been reported to be strongly expressed in mouse adult testis [4] and mkrn4 showed a slightly testis-biased expression in chicken.

Taken together, these results suggested that the ancestral $m k r n$ gene was preferentially expressed in the gonads in a primitive vertebrate, with conservation of expression pattern after duplication in fish and amphibians but partial relaxation of this specificity and possible extension of functions in birds and mammals.

$M k r n$ expression during early embryonic stages in fish largely reflected maternally stored mRNA expressed during oogenesis $[33,34]$, since mRNA levels were very high at very early stages (Figure 6). RNA levels then decreased throughout development, probably due to dilution effect through continuous cell division, and reached a very low level shortly after midblastula transition (MBT), the state of zygotic transcription activation. MBT takes place around stage 11 in medaka [35] and starts at cycle 10 corresponding to 512 cell stage in zebrafish [36] (between 128 cell stage and dome in our experiments). Maternal expression was observed for all three $m k r n$ genes in both medaka and zebrafish, suggesting here again an ancestral expression pattern. As observed in adults, no tissue-specific expression was observed in chicken embryos one day before hatching (Figure 6).

\section{Conclusion}

Through the identification of $m k r n 4$, a new member of the makorin gene family present in tetrapods and rayfinned fishes, we were able to gain some insights into the structural and functional evolution of this E3 ubiquitin ligase gene family. We could show that different evolutionary dynamics affect different $m k r n$ genes in vertebrates. While $m k r n 1$ and $m k r n 2$ were detected in all bony vertebrates studied, $m k r n 4$ was found to be absent from rat and mouse, two rodent murids, as well as from rabbit, a lagomorph. In addition, both Mkrn1 and Mkrn2 proteins have retained the ancestral arrangement of zinc fingers, with four $\mathrm{C} 3 \mathrm{H}$ motifs surrounding the RING domain responsible for the ubiquitin ligase activity. In contrast, Mkrn4 has lost several motifs early after its formation through duplication, and subsequent lineage-specific zinc finger loss was observed. Taken together, these observations suggest that Mkrn4 is subject to more relaxed evolutionary constraints than Mkrn1 and Mkrn2, with possible development of new general and lineage-specific functions. However, all Mkrn proteins contain the RING domain with adjacent C-terminal $\mathrm{C} 3 \mathrm{H}$ zinc finger, indicating that $\mathrm{E} 3$ ubiquitin ligase activity and interactions with nucleic acids might be properties common to all Makorin proteins. It appears therefore possible that Mkrn4 function, if still required in rat and mouse, might be performed by Mkrn1 or Mkrn2.

Through comparative genomics, we could find evidence that $m k r n 2$ and $m k r n 4$ have been duplicated together with other genes through a large-scale duplication. These genes are embedded in paralogons, i.e. in 


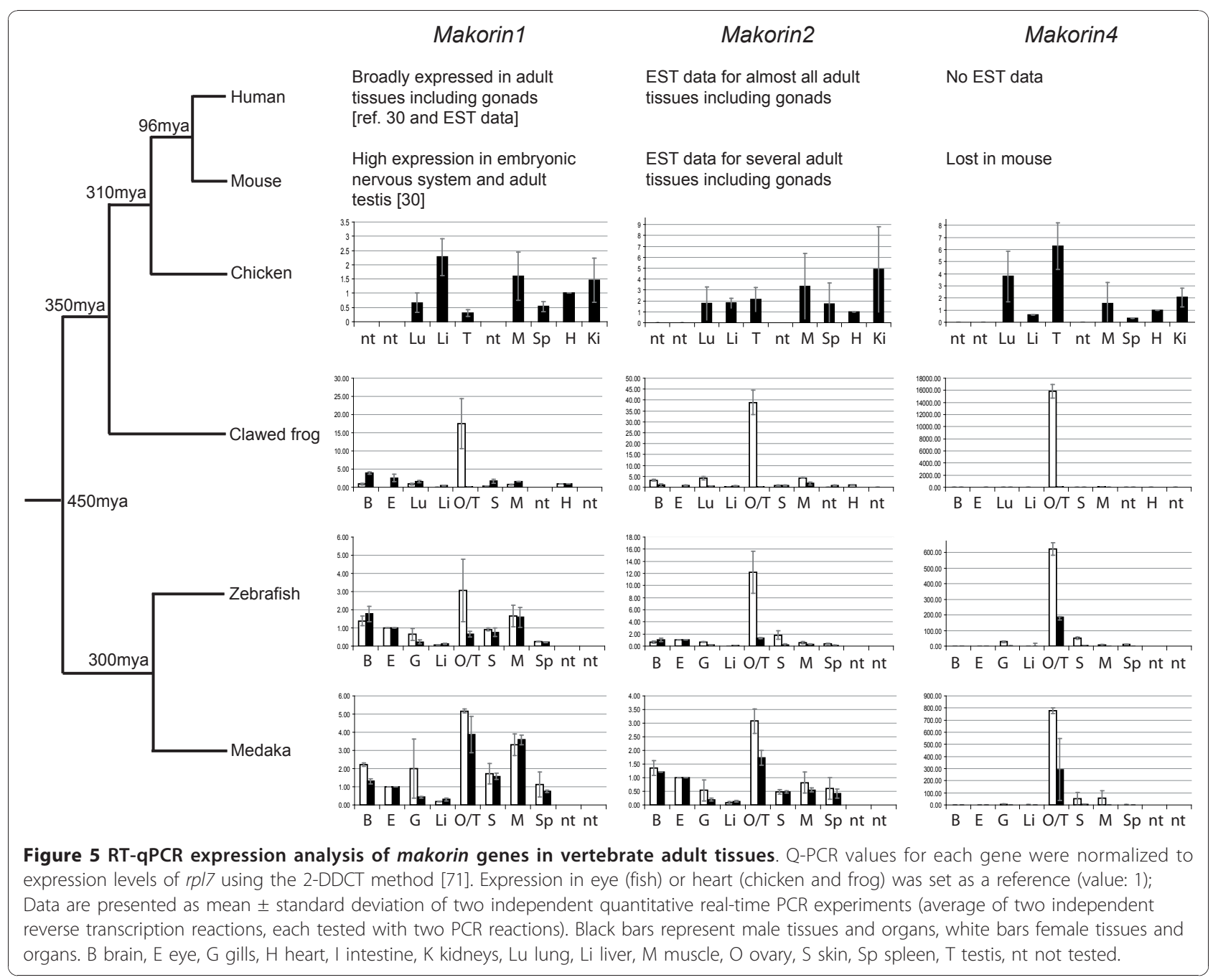

large syntenic chromosomal blocks of duplicated genes within a same genome. These paralogons were found in both tetrapods and ray-finned fish, tracing back their origin to at least 450 million years ago. We propose that $m k r n 2$ and $m k r n 4$ duplication was the result of either $1 \mathrm{R}$ or $2 \mathrm{R}$, the two events of genome duplication having taken place at the basis of the vertebrate lineage [12,26-28]. Both $1 \mathrm{R}$ and $2 \mathrm{R}$ arose before the split between cartilaginous and bony fish; one might therefore expect the presence of both $m k r n 2$ and $m k r n 4$ in the genome of elephant shark. However, only mkrn 2 was detected. This might be explained by secondary loss of $m k r n 4$ or incomplete coverage of the elephant shark genome. The molecular mechanism of formation of $m k r n 1$ remains elusive. Involvement of $1 \mathrm{R}$ or $2 \mathrm{R}$ appears possible (but we could not find any evidence for that) or of a more local event of segmental duplication. Conservation of intron position between $m k r n 1, m k r n 2$ and $m k r n 4$ (data not shown) suggests that retroposition was not involved.
After formation of $m k r n 1, m k r n 2$ and $m k r n 4$, the makorin gene family was shaped by lineage-specific retroposition-mediated duplications in vertebrates. Such retrogenes are formed by reverse transcription of generally mature mRNA molecules followed by integration of the formed cDNA into a new location of the genome. In mammals, retrogene formation is catalyzed by reverse transcriptase encoded by LINE-1 retrotransposons [37]. Retrogenes were detected for $m k r n 1$ and $m k r n 2$ in mammals but not in other vertebrates, and not for mkrn4. Most of the retrogenes are pseudogenes, but at least one of them, mkrn3, is functional and conserved in therian mammals. Hence, retroposition is an evolutionary mechanism of duplication and functional diversification in the $m k r n$ family. There is at the moment no satisfying explanation for the strong tendency of the $m k r n$ family to generate retrogenes. Germ cell expression, necessary for retrogene formation and transmission to the next generation, might predispose $m k r n$ genes to duplication through retroposition. Goncalves et al. [38] have 


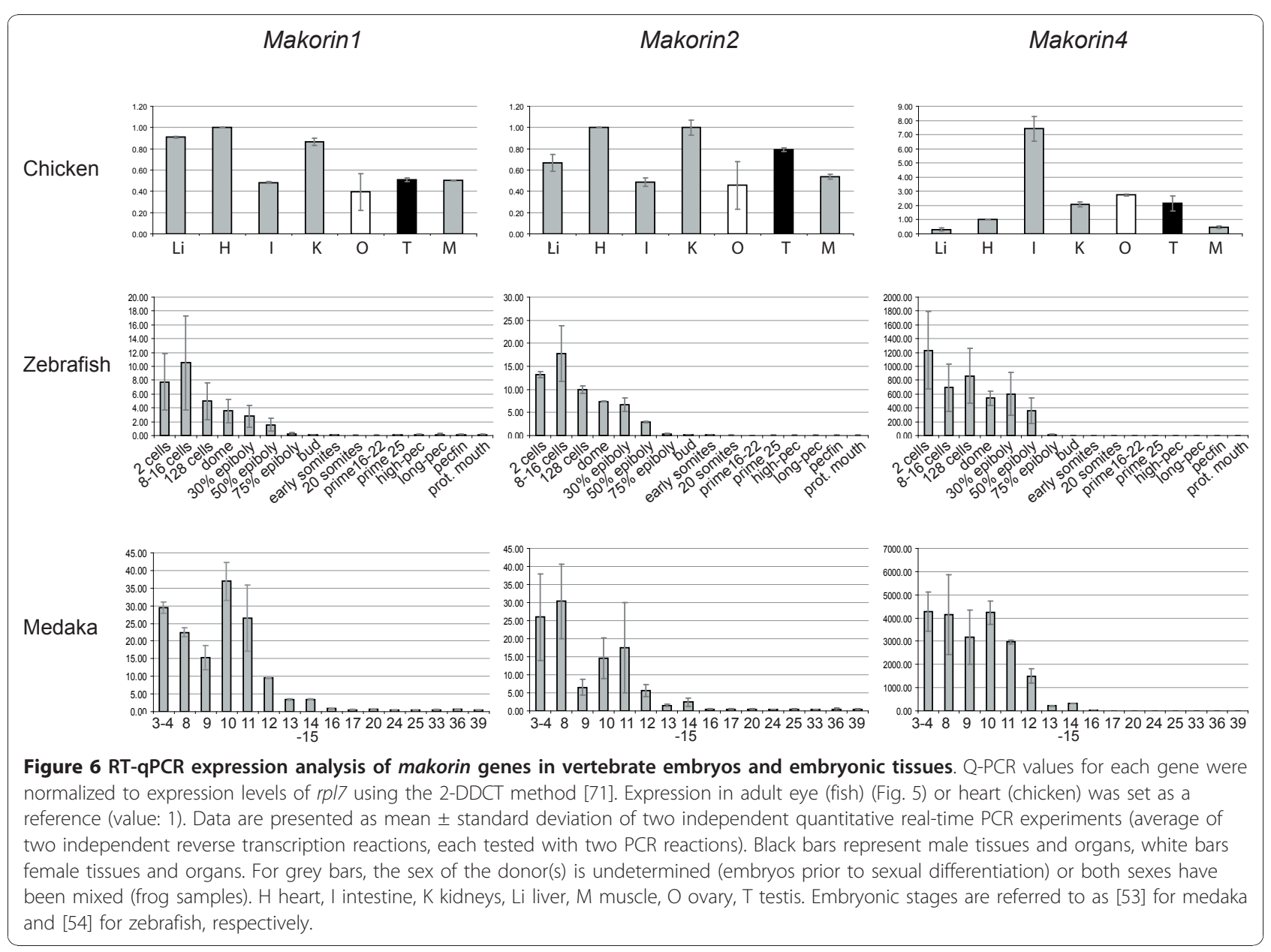

proposed that the gene expression level in the germ line is correlated with the number of retropseudogenes, since abundant mRNAs are more likely to be taken as a substrate by reverse-transcriptases. However, high $m k r n$ expression is not the only explanation, since no retrocopy was detected for $m k r n$ genes in amphibians and fish, in which they show a strong gonadal expression. Other factors might be involved, for example the affinity of expressed reverse transcriptases for mRNA molecules.

Finally, comparative expression analysis showed a strongly gonad-biased expression pattern for $m k r n 1$, $m k r n 2$ and $m k r n 4$ in medaka and zebrafish (ray-finned fishes) and amphibians, with particularly strong expression in ovary. Even if some $m k r n$ genes showed also preferential gonadal expression in chicken and mouse, expression was much less specific in birds and mammals. Maternal RNA was detected for all three genes in early medaka and zebrafish embryos. Taken together, these results suggest that the ancestral single-copy $m k r n$ gene was gonad-specific in primitive vertebrates, with a possible role in sexual development or gonad functions. This ancestral gene was also possibly maternally expressed and therefore potentially involved in fundamental processes such as fertilization, cell division or germ cell development [34].

Nothing is known concerning the functions of Mkrn proteins in fish and amphibian gonads and embryos, with the exception of a described role for Mkrn2 as a negative regulator of neurogenesis in Xenopus [18]. At the moment it is therefore difficult to propose a function for the ancestral vertebrate Mkrn protein, but results obtained for Mkrn1 suggest a link with the regulation of cell proliferation and apoptosis $[4,6]$. An involvement of the ubiquitin pathway in testis and spermatogenesis has been proposed for different organisms and testis-specific transcripts of different ubiquitin-interacting factors have been reported (for example [39-43]). Some data indicated an involvement of the ubiquitin pathway in the maintenance and development of ovarian germ cells in the fly [44] and the zebrafish [45] and during maturation of the ovary in mouse [46]. Several examples of maternally-expressed RING finger proteins with essential functions during development have been reported [47-51]. Multispecies comparative functional analysis of $m k r n$ genes is now required to better understand the evolution of this gene family in vertebrates. 


\section{Methods}

Fish

Platyfish (Xiphophorus maculatus, population Rio Jamapa WLC1274, closed colony stock derived from Jp163A), zebrafish (Danio rerio, strain AB/TU) and medaka (Oryzias latipes, strain Hd-rR) were kept under standard conditions at the PRECI aquarium facility of the IFR128 Biosciences Gerland-Lyon Sud (Lyon, France). Fish embryos were raised to the required stages of development in E3 embryo media [52] at $26^{\circ} \mathrm{C}$ for medaka and $28.5^{\circ} \mathrm{C}$ for zebrafish. Medaka and zebrafish embryos were staged according to Iwamatsu [53] and Kimmel et al. [54], respectively. Chicken samples (embryonic chicken, one day before hatching and male adult tissue) were provided by B. Pain (Institut de Génomique Fonctionnelle de Lyon, ENS de Lyon, France). Adult Xenopus laevis samples were gifts from F. Vollmar (Department of Cell and Developmental Biology, University of Würzburg, Germany) and C. Schultheis (Physiological Chemistry I, University of Würzburg, Germany). Experimental research reported here has been performed following internationally recognized guidelines (DDSV, Direction Départementale des Services Vétérinaires de Lyon, agreement number A 69387 0602).

\section{In silico analyses}

Protein and nucleotide sequences of $m k r n$ genes were identified using BLAST/BLAT searches against GenBank [55], the current Ensembl genome assemblies ([24] version 57, March 2010) of human (Homo sapiens), chimpanzee (Pan troglodytes), gorilla (Gorilla gorilla), orangutan (Pongo pygmaeus), macaque (Macaca mulatta), mouse lemur (Microcebus murinus), mouse (Mus musculus), guinea pig (Cavia porcellus), horse (Equus caballus), rabbit (Oryctolagus cuniculus), cow (Bos taurus), opossum (Monodelphis domestica), wallaby (Macropus eugenii), platypus (Ornithorhynchus anati$n u s)$, chicken (Gallus gallus), clawed frog (Xenopus tropicalis), zebrafish (Danio rerio), medaka (Oryzias latipes), green spotted pufferfish (Tetraodon nigroviridis), fugu (Takifugu rubripes), three-spined stickleback (Gasterosteus aculeatus), sea squirts (Ciona intestinalis and Ciona savignyi), the amphioxus genome (Branchiostoma floridae) [56] and the elephant shark genome (Callorhinchus milii) [57]. Automatic genome annotation was refined manually using EST data and related protein sequences with the help of FGENESH [58].

Nucleotide and protein sequences were loaded into BioEdit [59] and aligned using MUSCLE [60,61] and CLUSTALW2 [62]. Alignments were checked manually and ambiguously aligned regions were removed before phylogenetic analysis. Phylogenetic trees were calculated based on a 131 amino acid alignment including the RING domain and the downstream adjacent $\mathrm{C} 3 \mathrm{H}$ zinc finger. Maximum likelihood reconstruction was done using PhyML 3.0 [63,64], with 1000 bootstrap replicates under the LG model. Similar results were obtained using the neighbor-joining method as implemented in MEGA4 [65] with bootstrap values of 10.000 replicates. MEGA4 was also used to visualize phylogenetic trees. To establish syntenic relationships between genomes we used Genomicus [66], Synteny Database [67] and information provided by Ensembl Gene Trees [24]. Annotation of conserved protein motifs was performed with Motif Scan [68] and NCBI conserved domains [12].

$\mathrm{Ka} / \mathrm{Ks}$ calculation was peformed using $\mathrm{KaKsCalculator}$ under the MYN model [69] on pairwise nucleotide alignments done with LASTZ [70] and MUSCLE $[60,61]$. Indicated $\mathrm{Ka} / \mathrm{Ks}$ values are averages of values obtained for all possible pairwise comparisons.

\section{RT-qPCR expression analysis}

Total RNA was extracted from separated male and female tissues (brain, eyes, gills, heart, intestine, kidneys, lung, liver, muscle, ovary, skin, spleen and testis) and from whole embryos using the TRIReagent (Molecular Research Center, Inc.). After DNase treatment, reverse transcription was performed starting from $1 \mu \mathrm{g}$ of total RNA using the RevertAid First Strand Synthesis kit (Fermentas) and random hexamer primers. Real-time PCR was done on $2 \mu \mathrm{l}$ of dilutional series of cDNA starting from a dilution of factor 20 using IQTM Custom SYBR ${ }^{\circ}$ Green Supermix (Bio-Rad). PCR amplification was monitored with a CFX96 RealtimeSystem (Bio-Rad). After one incubation step at $95^{\circ} \mathrm{C}$ for $3 \mathrm{~min}$, the thermal cycling protocol was as follows: $95^{\circ} \mathrm{C}$ for 10 seconds, 55$62^{\circ} \mathrm{C}$ (primer-dependent annealing temperature) for 20 seconds and $72^{\circ} \mathrm{C}$ for 15 seconds during 40 cycles. Primers were designed manually and ordered from Invitrogen (Additional file 3). For each primer set, the efficiency of the PCR reaction was measured in duplicate on serial dilutions (factor 10) of the cDNA sample. Real-time PCR efficiencies $(E)$ for each reaction were calculated from the slope of the standard curve using the equation $E=10^{(-1 / \text { slope })}-1$ as implemented in the BioRad CFX manager V1.5 software, with $100 \%$ efficiency as an indicator of a robust assay. All results reported here are averages of two independent reverse transcription reactions, which have been each tested with two PCR reactions. For quantification, data were analyzed by the 2-DDCT method [71], by normalizing to the housekeeping gene rpl7 (ribosomal protein 17). Expression was normalized to eye tissue (fish) or heart (due to lack of eye samples for female frog and chicken). 


\section{Additional material}

\section{Additional file 1: Makorin sequence accession numbers. \\ Additional file 2: Genes used for synteny analysis. \\ Additional file 3: Real-time qPCR primers used}

\section{Acknowledgements}

This work was supported by a Ph.D. fellowship from the French Ministère de l'enseignement supérieur et de la recherche to $A B$ and by grants from the Agence Nationale de la Recherche (ANR), the Institut National de la Recherche Agronomique (INRA, département PHASE), the Centre National de la Recherche Scientifique (CNRS) and the Fondation pour la Recherche Médicale (FRM) (to JNV). We thank B. Pain for providing chicken samples, F. Vollmar and C. Schultheis for Xenopus samples and M. Sémon for helpful discussion on phylogenetic reconstruction and synteny analysis.

\section{Author details}

'Institut de Génomique Fonctionnelle de Lyon, Université de Lyon, Université Lyon 1, CNRS, INRA, Ecole Normale Supérieure de Lyon, Lyon, France. ${ }^{2}$ CIRAD, Upr20, Dept. Persyst, Campus International de Baillarguet, Montpellier, France.

\section{Authors' contributions}

$A B$ carried out the molecular genetic studies. $A B$ and JNV performed sequence analysis. $A B, H D C$ and JFB constructed the platyfish CDNA library. $A B$ and $A D$ identified and characterized mkrn4 in platyfish. $A B, D G A$ and JNV designed the study and wrote the paper. All authors read and approved the final manuscript.

Received: 29 July 2010 Accepted: 20 December 2010

Published: 20 December 2010

\section{References}

1. Deshaies RJ, Joazeiro CA: RING domain E3 ubiquitin ligases. Annu Rev Biochem 2009, 78:399-434.

2. Kim JH, Park SM, Kang MR, Oh SY, Lee TH, Muller MT, Chung IK: Ubiquitin ligase MKRN1 modulates telomere length homeostasis through a proteolysis of hTERT. Genes Dev 2005, 19:776-781.

3. Hall TM: Multiple modes of RNA recognition by zinc finger proteins. Curr Opin Struct Biol 2005, 15:367-373.

4. Gray TA, Hernandez L, Carey AH, Schaldach MA, Smithwick MJ, Rus K Marshall Graves JA, Stewart CL, Nicholls RD: The ancient source of a distinct gene family encoding proteins featuring RING and $\mathrm{C}(3) \mathrm{H}$ zincfinger motifs with abundant expression in developing brain and nervous system. Genomics 2000, 66:76-86

5. Ko A, Lee EW, Yang MR, Yeh JY, Moon JS, Song J: Mkrn1 induces the degradation of West Nile virus capsid protein by functioning as an E3 ligase. J Virol 2009, 84:426-436.

6. Lee EW, Lee MS, Camus S, Ghim J, Yang MR, Oh W, Ha NC, Lane DP, Song J: Differential regulation of $\mathrm{p} 53$ and p21 by MKRN1 E3 ligase controls cell cycle arrest and apoptosis. Embo J 2009, 28:2100-2113.

7. Salvatico J, Kim JH, Chung IK, Muller MT: Differentiation linked regulation of telomerase activity by Makorin-1. Mol Cell Biochem 2010, 342:241-250.

8. Omwancha J, Zhou XF, Chen SY, Baslan T, Fisher CJ, Zheng Z, Cai C, Shemshedini L: Makorin RING finger protein 1 (MKRN1) has negative and positive effects on RNA polymerase II-dependent transcription. Endocrine 2006, 29:363-373.

9. Shimada H, Shiratori T, Yasuraoka M, Kagaya A, Kuboshima M, Nomura F Takiguchi M, Ochiai T, Matsubara H, Hiwasa T: Identification of Makorin 1 as a novel SEREX antigen of esophageal squamous cell carcinoma. BMC Cancer 2009, 9:232

10. Yoshida N, Yano Y, Yoshiki A, Ueno M, Deguchi N, Hirotsune S: Identification of a new target molecule for a cascade therapy of polycystic kidney. Hum Cell 2003, 16:65-72

11. Knowles LM, Smith JW: Genome-wide changes accompanying knockdown of fatty acid synthase in breast cancer. BMC Genomics 2007, $8: 168$
12. National Center for Biotechnology Information Conserved domains. [http://www.ncbi.nlm.nih.gov/Structure/cdd/cdd.shtml].

13. Jong MT, Carey AH, Caldwell KA, Lau MH, Handel MA, Driscoll DJ, Stewart CL, Rinchik EM, Nicholls RD: Imprinting of a RING zinc-finger encoding gene in the mouse chromosome region homologous to the Prader-Willi syndrome genetic region. Hum Mol Genet 1999, 8:795-803.

14. Jong MT, Gray TA, Ji Y, Glenn CC, Saitoh S, Driscoll DJ, Nicholls RD: A novel imprinted gene, encoding a RING zinc-finger protein, and overlapping antisense transcript in the Prader-Willi syndrome critical region. Hum Mol Genet 1999, 8:783-793.

15. Kanber D, Giltay J, Wieczorek D, Zogel C, Hochstenbach R, Caliebe A, Kuechler A, Horsthemke B, Buiting K: A paternal deletion of MKRN3, MAGEL2 and NDN does not result in Prader-Willi syndrome. Eur J Hum Genet 2009, 17:582-590.

16. Gray TA, Wilson A, Fortin PJ, Nicholls RD: The putatively functional Mkrn 1p1 pseudogene is neither expressed nor imprinted, nor does it regulate its source gene in trans. Proc Natl Acad Sci USA 2006, 103:12039-12044.

17. Gray TA, Azama K, Whitmore K, Min A, Abe S, Nicholls RD: Phylogenetic conservation of the makorin-2 gene, encoding a multiple zinc-finger protein, antisense to the RAF1 proto-oncogene. Genomics 2001, 77:119-126.

18. Cheung WK, Yang PH, Huang QH, Chen Z, Chen SJ, Lin MCM, Kung HF: Identification of protein domains required for Makorin-2-mediated neurogenesis inhibition in Xenopus embryos. Biochem Biophys Res Commun 2010, 394:18-23.

19. Yang $P H$, Cheung WK, Peng Y, He ML, Wu GQ, Xie D, Jiang BH, Huang QH, Chen Z, Lin MC, Kung HF: Makorin-2 is a neurogenesis inhibitor downstream of phosphatidylinositol 3-kinase/Akt (PI3K/Akt) signal. J Biol Chem 2008, 283:8486-8495.

20. Tree families database. [http://www.treefam.org/cgi-bin/TFinfo.pl? $\mathrm{ac}=\mathrm{TF} 315108]$

21. Kasahara M, Naruse K, Sasaki S, Nakatani Y, Qu W, Ahsan B, Yamada T, Nagayasu Y, Doi K, Kasai $Y$, et al: The medaka draft genome and insights into vertebrate genome evolution. 2007, 447:714-719.

22. Jaillon O, Aury J-M, Brunet F, Petit J-L, Stange-Thomann N, Mauceli E, Bouneau L, Fischer C, Ozouf-Costaz C, Bernot A, et al: Genome duplication in the teleost fish Tetraodon nigroviridis reveals the early vertebrate proto-karyotype. 2004, 431:946-957.

23. Aparicio S, Chapman J, Stupka E, Putnam N, Chia J-m, Dehal P, Christoffels A, Rash S, Hoon S, Smit A, et al: Whole-genome shotgun assembly and analysis of the genome of Fugu rubripes. Science 2002, 297:1301-1310

24. Ensembl Genome Browser. [http://www.ensembl.org/index.html].

25. Venkatesh B, Kirkness EF, Loh YH, Halpern AL, Lee AP, Johnson J, Dandona N, Viswanathan LD, Tay A, Venter JC, et al: Survey sequencing and comparative analysis of the elephant shark (Callorhinchus milii) genome. PLOS Biol 2007, 5:e101.

26. Ohno S: Evolution by gene duplication New York: Springer Verlag; 1970

27. Van de Peer $Y$, Maere $S$, Meyer A: The evolutionary significance of ancient genome duplications. Nat Rev Genet 2009, 10:725-732.

28. Dehal P, Boore JL: Two rounds of whole genome duplication in the ancestral vertebrate. PLOS Biol 2005, 3:e314.

29. Ravi V, Lam K, Tay BH, Tay A, Brenner S, Venkatesh B: Elephant shark (Callorhinchus milii) provides insights into the evolution of Hox gene clusters in gnathostomes. Proc Natl Acad Sci USA 2009, 106:16327-16332.

30. Kaneko S, Aki I, Tsuda K, Mekada K, Moriwaki K, Takahata N, Satta Y: Origin and evolution of processed pseudogenes that stabilize functional Makorin1 mRNAs in mice, primates and other mammals. Genetics 2006, 172.2421-2429.

31. Vanin EF: Processed pseudogenes: Characteristics and evolution. Annu Rev Genet 1985, 19:253-272.

32. Tingaud-Sequeira A, Chauvigné F, Lozano J, Agulleiro MJ, Asensio E, Cerdà J: New insights into molecular pathways associated with flatfish ovarian development and atresia revealed by transcriptional analysis. BMC Genomics 2009, 10:434.

33. Abrams EW, Mullins MC: Early zebrafish development: it's in the maternal genes. Curr Opin Genet Dev 2009, 19:396-403.

34. Lindeman RE, Pelegri F: Vertebrate maternal-effect genes: Insights into fertilization, early cleavage divisions, and germ cell determinant localization from studies in the zebrafish. Mol Reprod Dev 2010, 77:299-313. 
35. Aizawaa K, Shimadab A, Naruseb K, Mitania H, Shimaa A: The medaka midblastula transition as revealed by the expression of the paternal genome. Gene Expr Patterns 2003, 3:43-47.

36. Kane DA, Kimmel CB: The zebrafish midblastula transition. Development 1993, 119:447-456

37. Esnault C, Maestre J, Heidmann T: Human LINE retrotransposons generate processed pseudogenes. Nat Genet 2000, 24:363-373.

38. Goncalves I, Duret L, Mouchiroud D: Nature and structure of human genes that generate retropseudogenes. Genome Res 2000, 10:672-678.

39. Luo J, Megee S, Dobrinski I: Asymmetric distribution of UCH-L1 in spermatogonia is associated with maintenance and differentiation of spermatogonial stem cells. J Cell Physio/ 2009, 220:460-468

40. Chianese R, Scarpa D, Berruti G, Cobellis G, Pierantoni R, Fasano S, Meccariello R: Expression and localization of the deubiquitinating enzyme UBPy in wobbler mouse testis during spermiogenesis. Gen Comp Endocrinol 2010, 166:289-295.

41. Nian H, Zhang W, Shi H, Zhao Q, Xie Q, Liao S, Zhang Y, Zhang Z, Wang C, Han C: Mouse RING finger protein Rnf133 is a testis-specific endoplasmic reticulum-associated E3 ubiquitin ligase. Cell Res 2008, 18:800-802.

42. Caldwell JC, Joiner ML, Sivan-Loukianova E, Eberl DF: The role of the RINGfinger protein Elfless in Drosophila spermatogenesis and apoptosis. Fly (Austin) 2008, 2:269-279.

43. Ribarski I, Lehavi O, Yogev L, Hauser R, B B-SM, Botchan A, Paz G, Yavetz H, Kleiman SE: USP26 gene variations in fertile and infertile men. Hum Reprod 2009, 24:477-484.

44. Chen D, Wang Q, Huang H, Xia L, Jiang X, Kan L, Sun Q, Chen D: Effetemediated degradation of Cyclin $A$ is essential for the maintenance of germline stem cells in Drosophila. Development 2009, 136:4133-4142.

45. Mo S, Song P, Lv D, Chen Y, Zhou W, Gong W, Zhu Z: Zebrafish z-otu, a novel Otu and Tudor domain-containing gene, is expressed in early stages of oogenesis and embryogenesis. Biochim Biophys Acta 2005 , 1732:1-7.

46. Gu YQ, Chen QJ, Gu Z, Shi Y, Yao YW, Wang J, Sun ZG, Tso JK: Ubiquitin carboxyl-terminal hydrolase L1 contributes to the oocyte selective elimination in prepubertal mouse ovaries. Sheng Li Xue Bao 2009, 61:175-184.

47. Kaido M, Wada H, Shindo M, Hayashi S: Essential requirement for RING finger E3 ubiquitin ligase Hakai in early embryonic development of Drosophila. Genes Cells 2009, 14:1067-1077.

48. Knowlton MN, Chan BM, Kelly GM: The zebrafish band 4.1 member Mir is involved in cell movements associated with gastrulation. Dev Biol 2003, 264:407-429.

49. Borden $\mathrm{KL}$, Lally JM, Martin SR, O'Reilly NJ, Etkin LD, Freemont PS: Novel topology of a zinc-binding domain from a protein involved in regulating early Xenopus development. EMBO J 1995, 14:5947-5956.

50. Bellini M, Lacroix J-C, Gall JG: A zinc-binding domain is required for targeting the maternal nuclear protein PwA33 to lampbrush chromosome loops. J Cell Biol 1995, 131:563-570.

51. Röttinger $E$, Besnardeau $L$, Lepage $T$ : Expression pattern of three putative RNA-binding proteins during early development of the sea urchin Paracentrotus lividus. Gene Expr Patterns 2006, 6:864-872.

52. Nüsslein-Vollhard C, Dahm R: Zebrafish: a practical approach Oxford University Press, USA; 2002.

53. Iwamatsu T: Stages of normal development in the medaka Oryzias latipes. Mech Dev 2004, 121:605-618.

54. Kimmel CB, Ballard WW, Kimmel SR, Ullmann B, Schilling TF: Stages of embryonic development of the zebrafish. Dev Dyn 1995, 203:253-310.

55. National Center for Biotechnology Information Basic Local Alignment Search Tool BLAST. [http://blast.ncbi.nlm.nih.gov/Blast.cgi].

56. Branchiostoma floridae v1.0. [http://genome.jgi-psf.org/Brafl1/Brafl1.home html].

57. Elephant shark genome project. [http://esharkgenome.imcb.a-star.edu.sg/].

58. SoftBerry FGENESH. [http://linux1.softberry.com/berry.phtml? topic=fgenesh\&group=programs\&subgroup=gfind].

59. Hall TA: BioEdit: a user-friendly biological sequence alignment editor and analysis program for Windows 95/98/NT. Nucl Acids Symp Ser 1999, 41:95-98.

60. Edgar RC: MUSCLE: a multiple sequence alignment method with reduced time and space complexity. BMC Bioinformatics 2004, 5:113.

61. Edgar RC: MUSCLE: multiple sequence alignment with high accuracy and high throughput. Nucleic Acids Res 2004, 32:1792-1797.
62. Larkin MA, Blackshields G, Brown NP, Chenna R, McGettigan PA, McWilliam H, Valentin F, Wallace IM, Wilm A, Lopez R, et al: ClustalW and ClustalX version 2. Bioinformatics 2007, 23:2947-2948.

63. Guindon S, Gascuel O: A simple, fast, and accurate algorithm to estimate large phylogenies by maximum likelihood. Syst Biol 2003, 52:696-704.

64. Guindon S, Dufayard JF, Lefort V, Anisimova M, Hordijk W, Gascuel O: New algorithms and methods to estimate maximum-likelihood phylogenies: assessing the performance of PhyML 3.0. Syst Biol 2010, 59:307-321.

65. Tamura K, Dudley J, Nei M, Kumar S: MEGA4: Molecular Evolutionary Genetics Analysis (MEGA) software version 4.0. Mol Biol Evol 2007, 24:1596-1599.

66. Muffato M, Louis A, Poisnel CE, Crollius HR: Genomicus: a database and a browser to study gene synteny in modern and ancestral genomes. Bioinformatics 2010, 26:1119-1121.

67. Catchen JM, Conery JS, Postlethwait JH: Automated identification of conserved synteny after whole genome duplication. Genome Res 2009, 19:1497-1505.

68. Motif Scan. [http://myhits.isb-sib.ch/cgi-bin/motif_scan]

69. Zhang Z, Li J, Zhao XQ, Wang J, Wong GK, Yu J: KaKs Calculator: Calculating $\mathrm{Ka}$ and $\mathrm{Ks}$ through model selection and model averaging. Genomics Proteomics Bioinformatics 2006, 4:259-263.

70. Miller Lab Software LASTZ alignment program. [http://www.bx.psu.edu/ miller_lab/].

71. Livak KJ, Schmittgen TD: Analysis of relative gene expression data using real-time auantitative PCR and the 2(-Delta Delta C(T)) method. Methods 2001, 25:402-408.

doi:10.1186/1471-2164-11-721

Cite this article as: Böhne et al:: The vertebrate makorin ubiquitin ligase gene family has been shaped by large-scale duplication and retroposition from an ancestral gonad-specific, maternal-effect gene. BMC Genomics 2010 11:721.

\section{Submit your next manuscript to BioMed Central and take full advantage of:}

- Convenient online submission

- Thorough peer review

- No space constraints or color figure charges

- Immediate publication on acceptance

- Inclusion in PubMed, CAS, Scopus and Google Scholar

- Research which is freely available for redistribution

Submit your manuscript at www.biomedcentral.com/submit
C Biomed Central 